\title{
Gluon Helicity Distribution at Small $x$
}

\section{Yuri V. Kovchegov*}

Department of Physics, The Ohio State University, Columbus, OH 43210, USA

E-mail: kovchegov.1@osu.edu

\section{Daniel Pitonyak}

Division of Science, Penn State University-Berks, Reading, PA 19610, USA

E-mail: dap67@psu.edu

\section{Matthew D. Sievert}

Theoretical Division, Los Alamos National Laboratory, Los Alamos, NM 87545, USA

E-mail: sievertmdelanl.gov

We determine the small- $x$ asymptotics of the gluon helicity distribution in a proton at leading order in perturbative QCD at large $N_{c}$. To achieve this, we begin by evaluating the dipole gluon helicity TMD at small $x$. We then construct and solve novel small- $x$ large- $N_{c}$ evolution equations for the operator related to the dipole gluon helicity TMD. Our main result is the small- $x$ asymptotics for the gluon helicity distribution:

$$
\Delta G \sim\left(\frac{1}{x}\right)^{\alpha_{h}^{G}} \text { with } \alpha_{h}^{G}=\frac{13}{4 \sqrt{3}} \sqrt{\frac{\alpha_{s} N_{c}}{2 \pi}} \approx 1.88 \sqrt{\frac{\alpha_{s} N_{c}}{2 \pi}} .
$$

We note that the power $\alpha_{h}^{G}$ is approximately 20\% lower than the corresponding power $\alpha_{h}^{q}$ for the small- $x$ asymptotics of the quark helicity distribution defined by

$$
\Delta q \sim\left(\frac{1}{x}\right)^{\alpha_{h}^{q}} \text { with } \alpha_{h}^{q}=\frac{4}{\sqrt{3}} \sqrt{\frac{\alpha_{s} N_{c}}{2 \pi}} \approx 2.31 \sqrt{\frac{\alpha_{s} N_{c}}{2 \pi}}
$$

found in our earlier work. 


\section{Introduction}

The main goal of this work is to determine the small- $x$ asymptotics of the quark and gluon helicity PDFs and TMDs. Below we will review our determination of the small- $x$ asymptotics of the quark helicity done in our earlier work, and then present the determination of the small- $x$ asymptotics for the gluon helicity distributions. This talk and these proceedings are mainly based on [1].

\section{Quark Helicity Distribution}

In [2], we derived the polarized small- $x$ evolution equations for the TMD quark helicity distribution [3],

$$
g_{1 L}^{q}\left(x, k_{T}^{2}\right)=\frac{1}{(2 \pi)^{3}} \frac{1}{2} \sum_{S_{L}} S_{L} \int d^{2} r d r^{-} e^{i x P^{+} r^{-}} e^{-i \underline{k} \cdot \underline{r}}\left\langle P, S_{L}\left|\bar{\psi}(0) \mathscr{U}[0, r] \frac{\gamma^{+} \gamma^{5}}{2} \psi(r)\right| P, S_{L}\right\rangle_{r^{+}=0},
$$

by relating it to a "polarized dipole amplitude" $G\left(x_{10}^{2}, z s\right)$, giving

$$
g_{1 L}^{q, S}\left(x, k_{T}^{2}\right)=\frac{8 N_{c}}{(2 \pi)^{6}} \sum_{f} \int_{\Lambda^{2} / s}^{1} \frac{d z}{z} \int d^{2} x_{01} d^{2} x_{0^{\prime} 1} e^{-i \underline{k} \cdot\left(\underline{x}_{01}-\underline{x}_{0^{\prime} 1}\right)} \frac{\underline{x}_{01} \cdot \underline{x}_{0^{\prime} 1}}{x_{01}^{2} x_{0^{\prime} 1}^{2}} G\left(x_{10}^{2}, z s=\frac{z}{x} Q^{2}\right)
$$

in the flavor-singlet case [4]. In the above and throughout this paper, we use light-front coordinates $x^{ \pm} \equiv \frac{1}{\sqrt{2}}\left(x^{0} \pm x^{3}\right)$, denote transverse vectors $\left(x_{\perp}^{1}, x_{\perp}^{2}\right)$ by $\underline{x}$ and their magnitudes by $x_{T} \equiv|\underline{x}|$, and indicate differences in transverse coordinates by the abbreviated notation $\underline{x}_{10} \equiv \underline{x}_{1}-\underline{x}_{0}$. The centerof-mass energy squared for the scattering process is $s$, the infrared (IR) transverse momentum cutoff is $\Lambda$, and $z$ is the fraction of the light-cone momentum of the dipole carried by the polarized (anti-)quark. As is well-known, the TMD (2.1) contains a process-dependent gauge link $\mathscr{U}[0, r]$. For specificity, in [2] we considered semi-inclusive deep inelastic scattering (SIDIS), although the resulting small- $x$ evolution equations also apply to the collinear quark helicity distribution, which is process independent.

The impact-parameter integrated polarized dipole amplitude is

$$
G\left(x_{10}^{2}, z s\right)=\int d^{2} b_{10} G_{10}(z s)
$$

with $\underline{b}_{10}=\left(\underline{x}_{1}+\underline{x}_{0}\right) / 2$. The polarized dipole scattering amplitude $G_{10}(z s)$ was defined as the polarized generalization of the forward dipole S-matrix in terms of Wilson lines [2]:

$$
\begin{aligned}
G_{10}(z s) & \equiv \frac{1}{2 N_{c}}\left\langle\left\langle\operatorname{tr}\left[V_{\underline{0}} V_{\underline{1}}^{p o l \dagger}\right]+\operatorname{tr}\left[V_{\underline{1}}^{p o l} V_{\underline{0}}^{\dagger}\right]\right\rangle\right\rangle(z s) \\
& \equiv \frac{z s}{2 N_{c}}\left\langle\operatorname{tr}\left[V_{\underline{0}} V_{\underline{1}}^{p o l \dagger}\right]+\operatorname{tr}\left[V_{\underline{1}}^{p o l} V_{\underline{0}}^{\dagger}\right]\right\rangle(z s),
\end{aligned}
$$

where the double-angle brackets are defined to scale out the center-of-mass energy zs between the polarized (anti)quark and the target. While the unpolarized Wilson lines in Eq. (2.4) are the standard eikonal gauge links (in the fundamental representation),

$$
V_{\underline{0}} \equiv V_{\underline{x}_{0}}[+\infty,-\infty] \equiv \mathscr{P} \exp \left[\operatorname{ig} \int_{-\infty}^{+\infty} d x^{-} A^{+}\left(x^{+}=0, x^{-}, \underline{x}_{0}\right)\right],
$$


the polarized Wilson lines $V_{\underline{1}}^{\text {pol }}$ are more complex operators [1]

$$
\left(V_{\underline{x}}^{p o l}\right)^{g}=\frac{i g p_{1}^{+}}{s} \int_{-\infty}^{+\infty} d x^{-} V_{\underline{x}}\left[+\infty, x^{-}\right] F^{12}\left(x^{+}=0, x^{-}, \underline{x}\right) V_{\underline{x}}\left[x^{-},-\infty\right]
$$

The expression for the polarized Wilson line in Eq. (2.6) is only due to the gluon interaction with the target (with the quark exchanges with the target to be included in the future work).

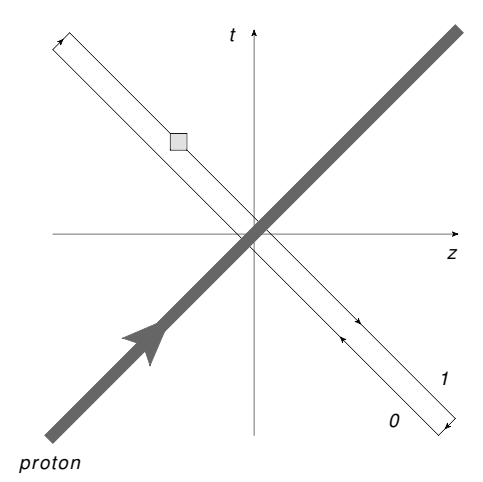

Figure 1: Pictorial representation of the polarized dipole operator in Eq. (2.7), with the square representing an insertion of the sub-eikonal operator $i g \varepsilon_{T}^{i j} \partial_{i} A^{j}$.

Employing Eq. (2.6) in Eq. (2.4) we can finally write down an explicit operator expression for the polarized dipole scattering amplitude (in $A^{-}=0$ gauge) illustrated in Fig. 1:

$$
G_{10}(z s) \equiv \frac{p_{1}^{+}}{2 N_{c}} \int_{-\infty}^{\infty} d x_{1}^{-}\left\langle\operatorname{tr}\left[V_{\underline{0}} V_{\underline{1}}\left[-\infty, x_{1}^{-}\right]\left(i g \varepsilon_{T}^{i j} \frac{\partial}{\partial\left(x_{1}\right)_{\perp}^{i}} A_{\perp}^{j}\left(x_{1}^{-}, \underline{x}_{1}\right)\right) V_{\underline{1}}\left[x_{1}^{-}, \infty\right]\right]+\text { c.c. }\right\rangle(z s) \text {. }
$$

To determine the small- $x$ asymptotics of the quark helicity distribution we need to find the high-energy limit of $G_{10}(z s)$. The latter is determined by deriving and solving the high-energy evolution equation for $G_{10}(z s)$. This equation is

$$
\begin{gathered}
G_{10}(z s)=G_{10}^{(0)}(z s)+\frac{\alpha_{s} N_{c}}{2 \pi^{2}} \int_{\frac{\Lambda^{2}}{s}}^{z} \frac{d z^{\prime}}{z^{\prime}} \int d^{2} x_{2}\left\{\left[\frac{1}{x_{21}^{2}}-\frac{\underline{x}_{21} \cdot \underline{x}_{20}}{x_{21}^{2} x_{20}^{2}}\right]\left\langle\left\langle\frac{1}{N_{c}^{2}} \operatorname{tr}\left[V_{\underline{0}} t^{a} V_{\underline{1}}^{\dagger} t^{b}\right]\left(U_{\underline{2}}^{p o l}\right)^{b a}+\text { c.c. }\right\rangle\right\rangle\left(z^{\prime} s\right)\right. \\
\left.+\frac{x_{10}^{2}}{x_{21}^{2} x_{20}^{2}}\left\langle\left\langle\frac{1}{N_{c}^{2}} \operatorname{tr}\left[V_{\underline{0}} t^{a} V_{\underline{1}}^{p o l \dagger} t^{b}\right]\left(U_{\underline{2}}\right)^{b a}-\frac{C_{F}}{N_{c}^{2}} \operatorname{tr}\left[V_{\underline{0}} V_{\underline{1}}^{p o l \dagger}\right]+\text { c.c. }\right\rangle\right\rangle\left(z^{\prime} s\right)\right\}
\end{gathered}
$$

and it is diagrammatically represented in Fig. 2. The equation resums powers of $\alpha_{s} \ln ^{2}(1 / x)$ in the quark helicity distribution: this is the double logarithmic approximation (DLA).

Equation (2.8) is not closed: the operator on the right-hand side is not the same as the operator on the left. To obtain a closed system of equations one has to invoke either the large- $N_{c}$ or the large$N_{c} \& N_{f}$ limits (the latter would also require the quark exchange contribution added to the polarized Wilson lines). In the large- $N_{c}$ limit, with DLA accuracy, the polarized evolution equations are [2, 4] 


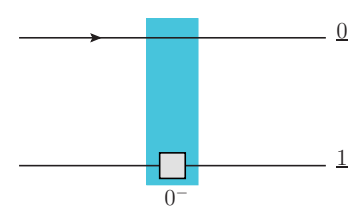

\section{— inhomogeneous term}
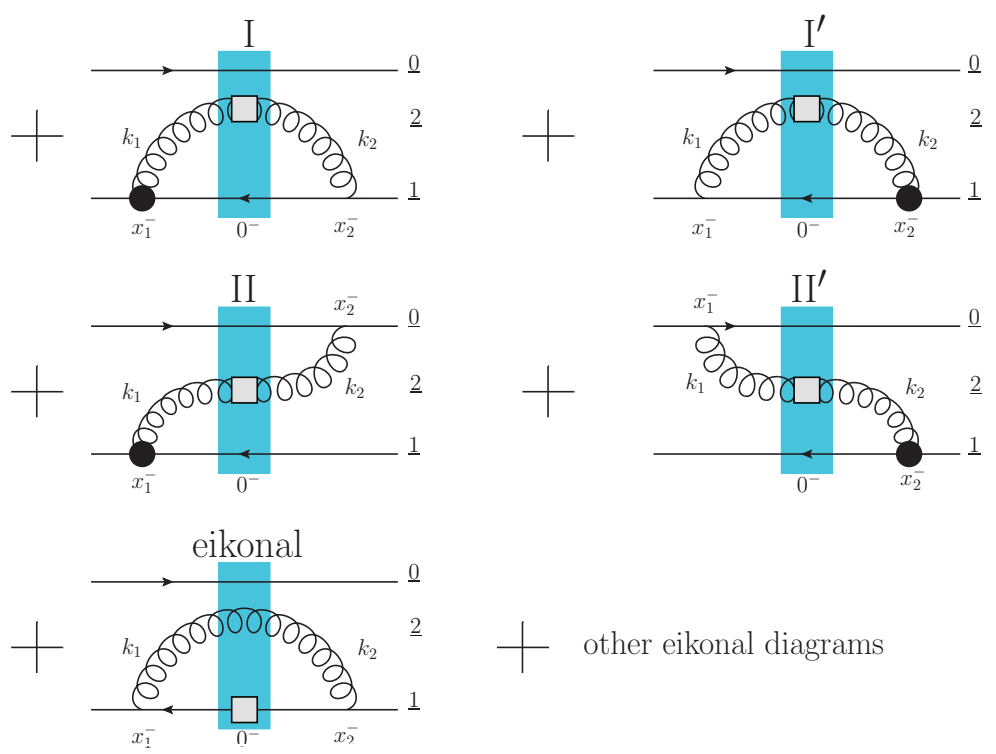

- $\quad$ other eikonal diagrams

Figure 2: Diagrams illustrating the evolution of the polarized dipole amplitude $G_{10}(z s)$. The blue band represents the classical fields (shock wave), the black vertex represents the sub-eikonal operator insertion from the center of (2.7), and the gray box represents the polarized Wilson line.

$$
\begin{gathered}
G\left(x_{10}^{2}, z s\right)=G^{(0)}\left(x_{10}^{2}, z s\right)+\frac{\alpha_{s} N_{c}}{2 \pi} \int_{\frac{1}{x_{10}^{2}}}^{z} \frac{d z^{\prime}}{z^{\prime}} \int_{\frac{1}{z^{\prime} s}}^{x_{10}^{2}} \frac{d x_{21}^{2}}{x_{21}^{2}}\left[\Gamma\left(x_{10}^{2}, x_{21}^{2}, z^{\prime} s\right)+3 G\left(x_{21}^{2}, z^{\prime} s\right)\right], \\
\Gamma\left(x_{10}^{2}, x_{21}^{2}, z^{\prime} s\right)=G^{(0)}\left(x_{10}^{2}, z^{\prime} s\right)+\frac{\alpha_{s} N_{c}}{2 \pi} \int_{\frac{1}{x_{10}}}^{z^{\prime}} \frac{d z^{\prime \prime}}{z^{\prime \prime}} \int_{\frac{1}{z^{\prime \prime} s}}^{\min \left[x_{10}^{2}, x_{21}^{2}{\frac{z^{\prime \prime}}{z^{\prime}}}^{\prime}\right.} \frac{d x_{32}^{2}}{x_{32}^{2}}\left[\Gamma\left(x_{10}^{2}, x_{32}^{2}, z^{\prime \prime} s\right)+3 G\left(x_{32}^{2}, z^{\prime \prime} s\right)\right],
\end{gathered}
$$

where $G^{(0)}$ are the initial conditions. The polarized dipole $G$ depends upon an auxiliary function $\Gamma$, termed the "neighbor dipole amplitude", in which further evolution is constrained by the lifetime of an adjacent dipole.

Equations (2.9) were solved numerically in [5] and analytically in [6] for the high-energy asymptotics yielding

$$
\begin{aligned}
G\left(x_{10}^{2}, z s\right) & =\frac{1}{3} G_{0}\left(z s x_{10}^{2}\right)^{\alpha_{h}^{q}} \\
\Gamma\left(x_{10}^{2}, x_{21}^{2}, z s\right) & =\frac{1}{3} G_{0}\left(z s x_{21}^{2}\right)^{\alpha_{h}^{q}}\left[4\left(\frac{x_{10}^{2}}{x_{21}^{2}}\right)^{\frac{\alpha_{h}^{q}}{4}}-3\right],
\end{aligned}
$$


where the exponent of the energy, known as the "quark helicity intercept" in analogy to the Pomeron intercept, is given by

$$
\alpha_{h}^{q}=\frac{4}{\sqrt{3}} \sqrt{\frac{\alpha_{s} N_{c}}{2 \pi}} \approx 2.31 \sqrt{\frac{\alpha_{s} N_{c}}{2 \pi}}
$$

This leads to Eq. (2) above. For further details, see the contribution of Matthew Sievert in these proceedings.

\section{Gluon Helicity Distribution}

The small- $x$ asymptotics of the gluon helicity can be determined in a similar way [1]. Start with the operator definition of the gluon dipole helicity TMD [7]

$$
\begin{aligned}
g_{1 L}^{G}\left(x, k_{T}^{2}\right)= & \frac{-2 i}{x P^{+}} \frac{1}{2} \sum_{S_{L}} S_{L} \int \frac{d \xi^{-} d^{2} \xi}{(2 \pi)^{3}} e^{i x P^{+} \xi^{-}-i \underline{k} \underline{\xi}} \\
& \times\left\langle P, S_{L}\left|\varepsilon_{T}^{i j} \operatorname{tr}\left[F^{+i}(0) \mathscr{U}^{[+]}[0, \xi] F^{+j}(\xi) \mathscr{U}^{[-]}[\xi, 0]\right]\right| P, S_{L}\right\rangle_{\xi^{+}=0}
\end{aligned}
$$

illustrated in Fig. 3.

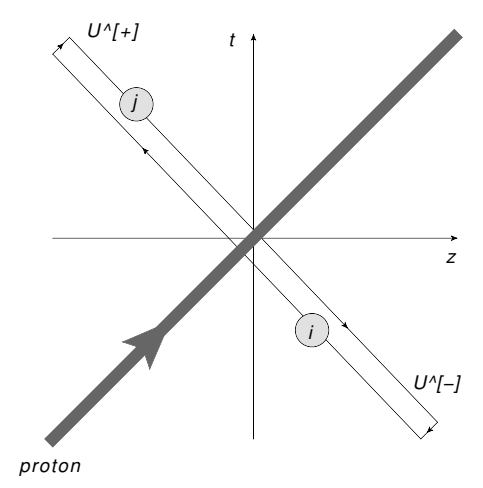

Figure 3: Pictorial representation of the gluon dipole helicity TMD in Eq. (3.1), with the circles representing insertions of $F^{+i}$ and $F^{+j}$.

It can be shown [6] that at small $x$ this TMD becomes

$$
g_{1 L}^{G d i p}\left(x, k_{T}^{2}\right)=\frac{-8 i N_{c}}{g^{2}(2 \pi)^{3}} \int d^{2} x_{10} e^{i k \cdot \underline{x}_{10}} k_{\perp}^{i} \varepsilon_{T}^{i j}\left[\int d^{2} b_{10} G_{10}^{j}\left(z s=\frac{Q^{2}}{x}\right)\right]
$$

where we have defined another dipole-like polarized operator

$$
G_{10}^{i}(z s) \equiv \frac{1}{2 N_{c}}\left\langle\operatorname{tr}\left[V_{\underline{\underline{ }}}\left(V_{\underline{1}}^{p o l \dagger}\right)_{\perp}^{i}\right]+\text { c.c. }\right\rangle(z s)
$$

with

$$
\left(V_{\underline{x}}^{p o l}\right)_{\perp}^{i} \equiv \int_{-\infty}^{+\infty} d x^{-} V_{\underline{x}}\left[+\infty, x^{-}\right]\left(\operatorname{ig}^{+} A_{\perp}^{i}(x)\right) V_{\underline{x}}\left[x^{-},-\infty\right] .
$$

The operator from Eq. (3.3) is shown in Fig. 4. 


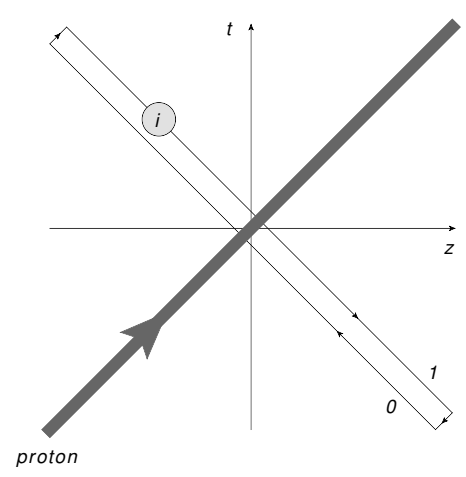

Figure 4: Pictorial representation of the new polarized dipole operator from Eq. (3.3), with the circle representing an insertion of the sub-eikonal transverse gluon field from Eq. (3.4).

After the integration over all impact parameters, the new polarized dipole amplitude is a vectorvalued function of $\underline{x}_{10}$ alone, allowing us to write the decomposition

$$
\int d^{2} b_{10} G_{10}^{i}(z s)=\left(x_{10}\right)_{\perp}^{i} G_{1}\left(x_{10}^{2}, z s\right)+\varepsilon_{T}^{i j}\left(x_{10}\right)_{\perp}^{j} G_{2}\left(x_{10}^{2}, z s\right) .
$$

The gluon dipole helicity TMD then becomes

$$
g_{1 L}^{G d i p}\left(x, k_{T}^{2}\right)=\frac{-N_{c}}{\alpha_{s} 2 \pi^{4}} \int d^{2} x_{10} e^{i \underline{k} \cdot \underline{x}_{10}}\left[1+x_{10}^{2} \frac{\partial}{\partial x_{10}^{2}}\right] G_{2}\left(x_{10}^{2}, z s=\frac{Q^{2}}{x}\right) .
$$

The DLA evolution equation for $G_{10}^{i}(z s)$ is illustrated diagrammatically in Fig. 5. The equation is

$$
\begin{aligned}
G_{10}^{i}(z s)= & G_{10}^{i(0)}(z s)+\frac{\alpha_{s} N_{c}}{2 \pi^{2}} \int_{\frac{\Lambda^{2}}{s}}^{z} \frac{d z^{\prime}}{z^{\prime}} \int d^{2} x_{2} \\
\times & \left\{\ln \frac{1}{x_{21} \Lambda} \varepsilon_{T}^{i j}\left[\frac{\left(x_{21}\right)_{\perp}^{j}}{x_{21}^{2}}-\frac{\left(x_{20}\right)_{\perp}^{j}}{x_{20}^{2}}\right]\left\langle\left\langle\frac{1}{2 N_{c}} \operatorname{tr}\left[V_{\underline{0}} V_{\underline{2}}^{p o l \dagger}\right]+\frac{1}{2 N_{c}} \operatorname{tr}\left[V_{\underline{2}}^{p o l} V_{\underline{1}}^{\dagger}\right]+\text { c.c. }\right\rangle\right\rangle\left(z^{\prime} s\right)\right\} \\
& +\frac{\alpha_{s} N_{c}}{2 \pi^{2}} \int_{\frac{\Lambda^{2}}{s}}^{z} \frac{d z^{\prime}}{z^{\prime}} \int \frac{d^{2} x_{2}}{x_{21}^{2}} \theta\left(x_{10}^{2}-x_{21}^{2}\right) \theta\left(x_{21}^{2}-\frac{1}{z^{\prime} s}\right)\left[G_{12}^{i}\left(z^{\prime} s\right)-\Gamma_{10,21}^{i}\left(z^{\prime} s\right)\right]
\end{aligned}
$$

where the have introduced the "neighbor" dipole amplitude $\Gamma^{i}$ by analogy with the quark helicity evolution case.

Equation (3.7) again does not close. To obtain a closed system of equations we take the large$N_{c}$ limit. Remembering that we are interested in $G_{2}$ to find the gluon helicity TMD in Eq. (3.6), we 

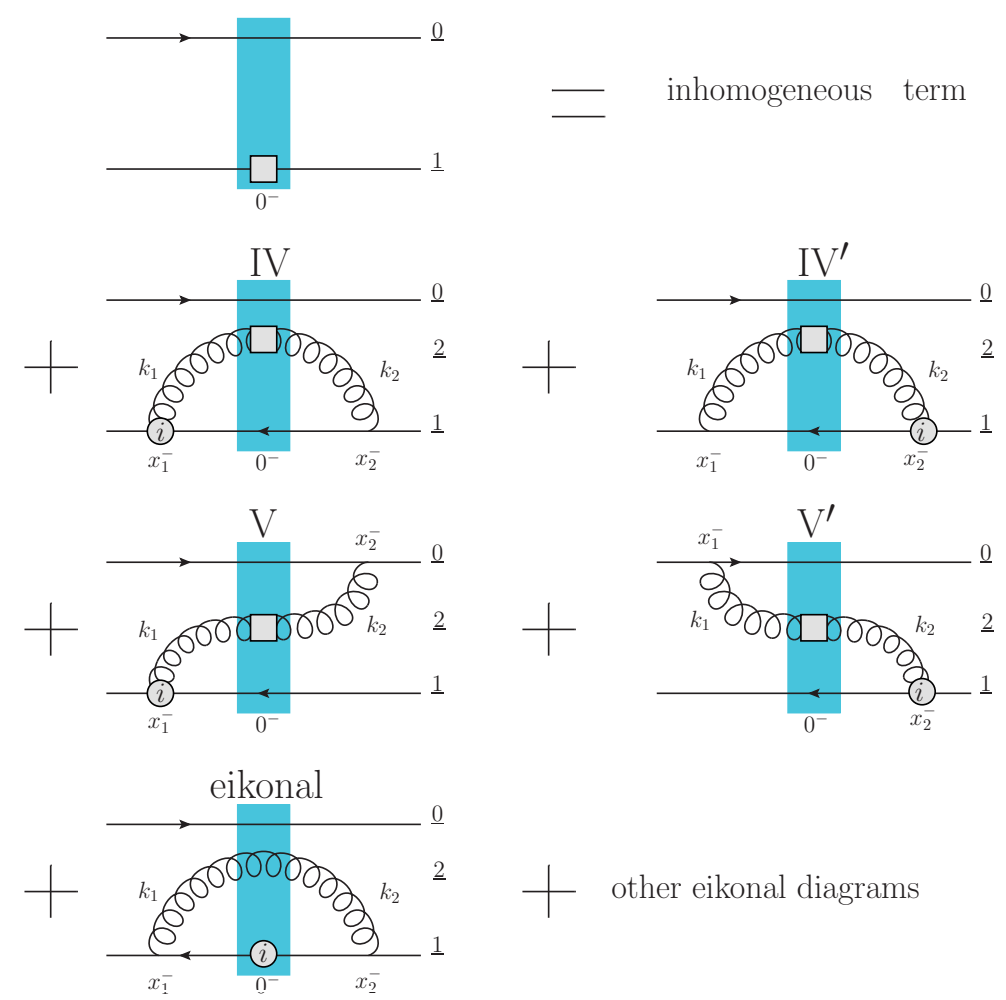

$+\quad$ other eikonal diagrams

Figure 5: Diagrams illustrating the evolution of the polarized dipole amplitude for the dipole gluon helicity distribution. The blue band represents the classical gluon fields (shock wave), the vertex $(i)$ denotes the sub-eikonal operator insertion, and the gray box represents the polarized Wilson line.

project out the $G_{2}$ component to write

$$
\begin{aligned}
& G_{2}\left(x_{10}^{2}, z s\right)=G_{2}^{(0)}\left(x_{10}^{2}, z s\right)+\frac{\alpha_{s} N_{c}}{2 \pi^{2}} \int_{\frac{\Lambda^{2}}{s}}^{z} \frac{d z^{\prime}}{z^{\prime}} \int d^{2} x_{2} \ln \frac{1}{x_{21} \Lambda} \frac{x_{10} \cdot \underline{x}_{21}}{x_{10}^{2} x_{21}^{2}}\left[\Gamma_{g e n}\left(x_{20}^{2}, x_{21}^{2}, z^{\prime} s\right)+G\left(x_{21}^{2}, z^{\prime} s\right)\right] \\
& -\frac{\alpha_{s} N_{c}}{2 \pi^{2}} \int_{\frac{\Lambda^{2}}{s}}^{z} \frac{d z^{\prime}}{z^{\prime}} \int d^{2} x_{2} \ln \frac{1}{x_{21} \Lambda} \frac{x_{10} \cdot \underline{x}_{20}}{x_{10}^{2} x_{20}^{2}}\left[\Gamma_{g e n}\left(x_{20}^{2}, x_{21}^{2}, z^{\prime} s\right)+\Gamma_{g e n}\left(x_{21}^{2}, x_{20}^{2}, z^{\prime} s\right)\right] \\
& -\frac{\alpha_{s} N_{c}}{2 \pi} \int_{\frac{1}{x_{10}^{2}}}^{z} \frac{d z^{\prime}}{z^{\prime}} \int_{\frac{1}{z^{\prime} s}}^{x_{10}^{2}} \frac{d x_{21}^{2}}{x_{21}^{2}} \Gamma_{2}\left(x_{10}^{2}, x_{21}^{2}, z^{\prime} s\right) \\
& \Gamma_{2}\left(x_{10}^{2}, x_{21}^{2}, z^{\prime} s\right)=G_{2}^{(0)}\left(x_{10}^{2}, z^{\prime} s\right)+\frac{\alpha_{s} N_{c}}{2 \pi^{2}} \int_{\frac{\Lambda^{2}}{s}}^{z^{\prime}} \frac{d z^{\prime \prime}}{z^{\prime \prime}} \int d^{2} x_{3} \ln \frac{1}{x_{31} \Lambda} \frac{x_{10} \cdot \underline{x}_{31}}{x_{10}^{2} x_{31}^{2}}\left[\Gamma_{g e n}\left(x_{30}^{2}, x_{31}^{2}, z^{\prime \prime} s\right)+G\left(x_{31}^{2}, z^{\prime} s\right)\right] \\
& -\frac{\alpha_{s} N_{c}}{2 \pi^{2}} \int_{\frac{\Lambda^{2}}{s}}^{z^{\prime}} \frac{d z^{\prime \prime}}{z^{\prime \prime}} \int d^{2} x_{3} \ln \frac{1}{x_{31} \Lambda} \frac{x_{10} \cdot \underline{x}_{30}}{x_{10}^{2} x_{30}^{2}}\left[\Gamma_{g e n}\left(x_{30}^{2}, x_{31}^{2}, z^{\prime \prime} s\right)+\Gamma_{g e n}\left(x_{31}^{2}, x_{30}^{2}, z^{\prime \prime} s\right)\right] \\
& -\frac{\alpha_{s} N_{c}}{2 \pi} \int_{\frac{1}{x_{10}^{2}}}^{z^{\prime}} \frac{d z^{\prime \prime}}{z^{\prime \prime}} \int_{\frac{1}{z^{\prime \prime}}}^{\min \left[x_{10}^{2}, x_{2}^{2} 1 \frac{z^{\prime}}{z^{\prime \prime}}\right]} \frac{d x_{31}^{2}}{x_{31}^{2}} \Gamma_{2}\left(x_{10}^{2}, x_{31}^{2}, z^{\prime \prime} s\right) .
\end{aligned}
$$


We have defined an impact-parameter integrated amplitude $\Gamma_{g e n}$ by

$$
\Gamma_{g e n}\left(x_{20}, x_{21}, z^{\prime} s\right)=\theta\left(x_{20}-x_{21}\right) \Gamma\left(x_{20}, x_{21}, z^{\prime} s\right)+\theta\left(x_{21}-x_{20}\right) G\left(x_{20}, z^{\prime} s\right) .
$$

This function can be easily found using the analytic solution (2.10) for the asymptotics of $G$ and $\Gamma$ at high energies.

Equations (3.8) can be solved analytically [1]. The result it

$$
\begin{aligned}
& G_{2}\left(x_{10}^{2}, z s\right) \approx-\frac{19}{32 \sqrt{3}} \sqrt{\frac{\alpha_{s} N_{c}}{2 \pi}} G_{0} \ln \frac{1}{x_{10} \Lambda}\left(z s x_{10}^{2}\right)^{\frac{13}{4 \sqrt{3}} \sqrt{\frac{\alpha_{s} N_{c}}{2 \pi}}}, \\
& \Gamma_{2}\left(x_{10}^{2}, x_{21}^{2}, z^{\prime} s\right) \approx-\frac{1}{2 \sqrt{3}} \sqrt{\frac{\alpha_{s} N_{c}}{2 \pi}} G_{0} \ln \frac{1}{x_{10} \Lambda} {\left[\left(z^{\prime} s x_{10}^{2}\right)^{\frac{4}{\sqrt{3}} \sqrt{\frac{\alpha_{s} N_{c}}{2 \pi}}}\left(z^{\prime} s x_{21}^{2}\right)^{-\frac{\sqrt{3}}{4} \sqrt{\frac{\alpha_{s} N_{c}}{2 \pi}}}\right.} \\
&\left.+\frac{3}{16}\left(z^{\prime} s x_{21}^{2}\right)^{\frac{4}{\sqrt{3}} \sqrt{\frac{\alpha_{s} N_{c}}{2 \pi}}}\left(z^{\prime} s x_{10}^{2}\right)^{-\frac{\sqrt{3}}{4} \sqrt{\frac{\alpha_{s} N_{c}}{2 \pi}}}\right] .
\end{aligned}
$$

The asymptotic solution (3.10a) for the polarized dipole amplitude $G_{2}$ is the central result of this work. Substituting the solution Eq. (3.10a) into Eq. (3.6) yields the small- $x$ asymptotics of the dipole gluon helicity distribution:

$$
g_{1 L}^{G d i p}\left(x, k_{T}^{2}\right) \sim G_{2}\left(x_{10}^{2}, z s=\frac{Q^{2}}{x}\right) \sim\left(\frac{1}{x}\right)^{\alpha_{h}^{G}}
$$

with the gluon helicity intercept

$$
\alpha_{h}^{G}=\frac{13}{4 \sqrt{3}} \sqrt{\frac{\alpha_{s} N_{c}}{2 \pi}} \approx 1.88 \sqrt{\frac{\alpha_{s} N_{c}}{2 \pi}} .
$$

We conclude that (cf. Eq. (1))

$$
\Delta G\left(x, Q^{2}\right) \sim\left(\frac{1}{x}\right)^{\alpha_{h}^{G}} \sim\left(\frac{1}{x}\right)^{\frac{13}{4 \sqrt{3}} \sqrt{\frac{\alpha_{s} N_{c}}{2 \pi}}} \sim\left(\frac{1}{x}\right)^{1.88 \sqrt{\frac{\alpha_{s} N_{c}}{2 \pi}}} .
$$

\section{Phenomenology of the Gluon Spin at Small $x$}

In this section we give an estimate for the gluon spin $S_{G}$,

$$
S_{G}\left(Q^{2}\right)=\int_{0}^{1} d x \Delta G\left(x, Q^{2}\right),
$$

based on our gluon helicity intercept (3.12).

In order to calculate $S_{G}$, we need input for the gluon helicity PDF $\Delta G\left(x, Q^{2}\right)$, and we focus here on the fit from DSSV14 [8]. We proceed through a simple approach, which we also employed in Ref. [5] for an estimate of the quark spin, and leave a more rigorous phenomenological study for future work. First, we attach a curve $\Delta \tilde{G}\left(x, Q^{2}\right)=N x^{-\alpha_{h}^{G}}$ (with $\alpha_{h}^{G}$ given in (3.12)) to the DSSV14 result for $\Delta G\left(x, Q^{2}\right)$ at a particular small- $x$ point $x_{0}$. We fix the normalization $N$ by requiring $\Delta \tilde{G}\left(x_{0}, Q^{2}\right)=\Delta G\left(x_{0}, Q^{2}\right)$. Then we calculate the truncated integral

$$
S_{G}^{\left[X_{\min }\right]}\left(Q^{2}\right) \equiv \int_{x_{\min }}^{1} d x \Delta G\left(x, Q^{2}\right)
$$



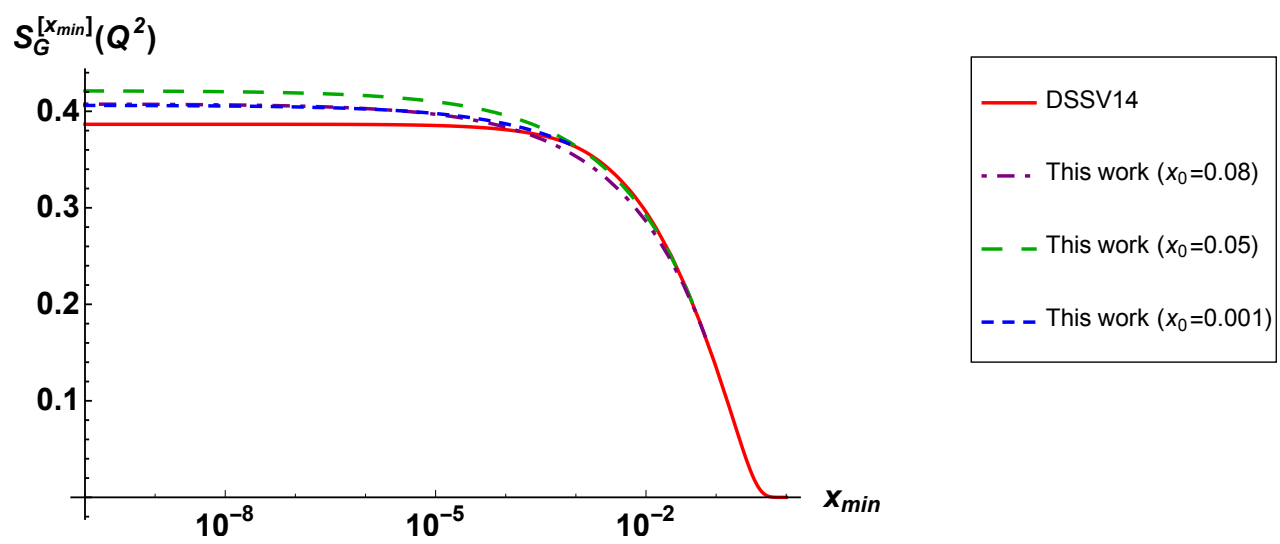

Figure 6: Plot of $S_{G}^{\left[x_{\text {min }}\right]}\left(Q^{2}\right)$ vs. $x_{\text {min }}$ at $Q^{2}=10 \mathrm{GeV}^{2}$. The solid curve is from DSSV14 [8]. The dotdashed, long-dashed, and short-dashed curves are from various small- $x$ modifications of $\Delta G\left(x, Q^{2}\right)$ at $x_{0}=$ $0.08,0.05,0.001$, respectively, using our gluon helicity intercept (see the text for details).

of the modified gluon helicity PDF

$$
\Delta G_{m o d}\left(x, Q^{2}\right) \equiv \theta\left(x-x_{0}\right) \Delta G\left(x, Q^{2}\right)+\theta\left(x_{0}-x\right) \Delta \tilde{G}\left(x, Q^{2}\right)
$$

for different $x_{0}$ values. The results are shown in Fig. 6 for $Q^{2}=10 \mathrm{GeV}^{2}$ and $\alpha_{s} \approx 0.25$, in which case $\alpha_{h}^{G} \approx 0.65$. We see that the small- $x$ evolution of $\Delta G\left(x, Q^{2}\right)$ gives about a $5 \div 10 \%$ increase to the gluon spin, depending on where in $x$ the effects set in and on the parameterization of the gluon helicity PDF at higher $x$. Again we emphasize that the first principles results of this work (along with that for the quark $[2,5,6]$ ) can be included in future extractions of helicity PDFs, especially once the present large- $N_{c}$ approximation is relaxed, which will provide strong constraints on the small- $x$ behavior of the quark and gluon spin.

Saturation effects may also impact the amount of spin carried by small- $x$ quarks and gluons. The small- $x$ asymptotics of $\Delta G$ found here and the small- $x$ asymptotics of $\Delta q$ found in $[2,5,6]$ are such that $x \Delta G \rightarrow 0$ and $x \Delta q \rightarrow 0$ as $x \rightarrow 0$. Hence the helicity PDFs will not violate unitarity at small $x$. However, as one can see from the helicity evolution equations including (LLA) saturation effects, as derived in $[2,4]$, saturation would completely suppress the small- $x$ evolution of helicity PDFs, making the effective $\alpha_{h}^{q}$ and $\alpha_{h}^{G}$ zero in the saturation region (cf. [9] for the flavor nonsinglet unpolarized quark distribution). Therefore, a very small amount of the proton spin should reside in the saturation region. This observation can become an important component of the future small- $x$ helicity PDF phenomenology.

\section{Acknowledgments}

This material is based upon work supported by the U.S. Department of Energy, Office of Science, Office of Nuclear Physics under Award Number DE-SC0004286 (YK), within the framework of the TMD Topical Collaboration (DP), and DOE Contract No. DE-AC52-06NA25396 (MS). MS received additional support from the U.S. Department of Energy, Office of Science under the DOE Early Career Program. 


\section{References}

[1] Y. V. Kovchegov, D. Pitonyak and M. D. Sievert, Small-x Asymptotics of the Gluon Helicity Distribution, 1706.04236.

[2] Y. V. Kovchegov, D. Pitonyak and M. D. Sievert, Helicity Evolution at Small-x, JHEP 01 (2016) 072, [1511.06737].

[3] P. J. Mulders and R. D. Tangerman, The Complete tree level result up to order $1 / Q$ for polarized deep inelastic leptoproduction, Nucl. Phys. B461 (1996) 197-237, [hep-ph/9510301].

[4] Y. V. Kovchegov, D. Pitonyak and M. D. Sievert, Helicity Evolution at Small x: Flavor Singlet and Non-Singlet Observables, Phys. Rev. D95 (2017) 014033, [1610 . 06197$].$

[5] Y. V. Kovchegov, D. Pitonyak and M. D. Sievert, Small-x asymptotics of the quark helicity distribution, Phys. Rev. Lett. 118 (2017) 052001, [1610.06188].

[6] Y. V. Kovchegov, D. Pitonyak and M. D. Sievert, Small-x Asymptotics of the Quark Helicity Distribution: Analytic Results, 1703.05809.

[7] C. J. Bomhof, P. J. Mulders and F. Pijlman, The Construction of gauge-links in arbitrary hard processes, Eur. Phys. J. C47 (2006) 147-162, [hep-ph/ 0601171$].$

[8] D. de Florian, R. Sassot, M. Stratmann and W. Vogelsang, Evidence for polarization of gluons in the proton, Phys. Rev. Lett. 113 (2014) 012001, [1404.4293].

[9] K. Itakura, Y. V. Kovchegov, L. McLerran and D. Teaney, Baryon stopping and valence quark distribution at small x, Nucl. Phys. A730 (2004) 160-190, [hep-ph/0305332]. 